\title{
Exact Solution of the Dirac Equation with a Central Potential
}

\author{
E. J. Kanellopoulos, Th. V. Kanellopoulos, and K. Wildermuth
}

Institut für Theoretische Physik der Universität Tübingen

Received March 24, 1972

Abstract. The exact solution of the Dirac equation with a central potential, in the semi-relativistic approximation, is derived and formulae for phase shifts and eigenvalue equations are given.

\section{Introduction}

The integro-iteration method, introduced in Ref. [1] is applied to the solution of the Dirac's coupled radial equations. The solutions are obtained in a form similar to that of the Schrödinger equation [2], i.e., in simple series which converge strongly when the following restrictions are imposed on the potential $V(r)$ :

$$
V_{r \rightarrow 0}(r) \rightarrow r^{-\beta} \beta \leqq 1
$$

and

$$
\int_{a}^{\infty} V(r) d r<\infty \text { for } 0<a<\infty
$$

Condition $(1 \mathrm{~b})$ excludes the Coulomb potential, but in this case the solutions are already known $[3,4]$. On the other hand in cases with a screened or modified Coulomb potential [5] the method is applicable and one can get results to any desired accuracy.

\section{Formulation}

In semi-relativistic approximation the Dirac equation with central potential, after separation of the angular part, [3], is reduced to a system of two coupled radial equations [5];

$$
\begin{gathered}
(E+V+m) F_{v}+\frac{d G_{v}}{d r}-\frac{v}{r} G_{v}=0 \\
-(E+V-m) G_{v}+\frac{d F_{v}}{d r}+\frac{v+2}{r} F_{v}=0 .
\end{gathered}
$$


Here we use the same notation as in Ref. [5], but for simplicity we have put $\hbar=c=1$, and $v=l$ for $j=l-\frac{1}{2}$ and $v=-l-1$ for $j=l+\frac{1}{2}$. If we put

$$
\begin{aligned}
G_{v} & =\frac{g_{v}}{r} \\
F_{v} & =\frac{f_{v}}{r}
\end{aligned}
$$

we obtain the more symmetrical form:

$$
\begin{gathered}
(E+V+m) f_{v}+g_{v}^{\prime}-\frac{v+1}{r} g_{v}=0 \\
-(E+V-m) g_{v}+f_{v}^{\prime}+\frac{v+1}{r} f_{v}=0 .
\end{gathered}
$$

For $V=0$ the solutions of (4) are readily obtainable and are expressed in terms of Bessel functions if $E^{2}-m^{2}=k^{2}>0$ and modified Bessel functions if $E^{2}-m^{2}=-\kappa^{2}<0$.

Let $u_{1}$ and $u_{2}$ be two independent solutions of (4), with $V=0$, regular respectively irregular at the origin, corresponding to $g_{v}^{0}$ and $v_{1}$ and $v_{2}$ those corresponding to $f_{v}^{0}$. We normalize them in such a way that

$$
\operatorname{det}\left|\begin{array}{ll}
u_{1} & u_{2} \\
v_{1} & v_{2}
\end{array}\right|=1^{1}
$$

Next we look for a solution of (4) in the form:

$$
\begin{aligned}
& g_{v}(r)=C_{1}(r) u_{1}(r)+C_{2}(r) u_{2}(r) \\
& f_{v}(r)=C_{1}(r) v_{1}(r)+C_{2}(r) v_{2}(r)
\end{aligned}
$$

where $C_{1}(r)$ and $C_{2}(r)$ are functions to be specified, such that (6) are solutions of Eqs. (4). Using Lagrange's method of undetermined coefficients, and taking into account (5) we find:

$$
\begin{aligned}
& C_{1}^{\prime}(r)=-C_{1} V\left(u_{1} u_{2}+v_{1} v_{2}\right)-C_{2} V\left(u_{2}^{2}+v_{2}^{2}\right) \\
& C_{2}^{\prime}(r)=+C_{2} V\left(u_{1} u_{2}+v_{1} v_{2}\right)+C_{1} V\left(u_{1}^{2}+v_{1}^{2}\right) .
\end{aligned}
$$

Applying the integro-iteration method [1] we find the general solution of $(7)^{2}$ :

$$
\begin{aligned}
& C_{1}(r)=\lambda_{1} e^{-f(r)} \Phi_{1}\left(\begin{array}{c}
r \\
a, a
\end{array}\right)-\lambda_{2} e^{-f(r)} \int_{a}^{r} A_{22} e^{2 f\left(r^{\prime}\right)} \Phi_{2}\left(\begin{array}{c}
r^{\prime} \\
a, a
\end{array}\right) d r^{\prime} \\
& C_{2}(r)=\lambda_{2} e^{f(r)} \Phi_{2}\left(\begin{array}{c}
r \\
a, a
\end{array}\right)+\lambda_{1} e^{f(r)} \int_{a}^{r} A_{11} e^{-2 f\left(r^{\prime}\right)} \Phi_{1}\left(\begin{array}{c}
r^{\prime} \\
a, a
\end{array}\right) d r^{\prime}
\end{aligned}
$$

1 For explicit expressions of $u_{j}, v_{j}(j=1,2)$ for every case see Appendix.

2 We use the same notation as in Ref. [1]. 
where $\lambda_{1}, \lambda_{2}$ are arbitrary constants,

$$
\begin{aligned}
& A_{11}=V\left[u_{1}^{2}+v_{1}^{2}\right] \\
& A_{22}=V\left[u_{2}^{2}+v_{2}^{2}\right] \\
& A_{12}=V\left[u_{1} u_{2}+v_{1} v_{2}\right] \\
& f(r)=\int_{a}^{r} A_{12} d r^{\prime}
\end{aligned}
$$

and

$$
\begin{aligned}
& \Phi_{1}\left(\begin{array}{c}
r \\
a, a
\end{array}\right)=1-\int_{a}^{r} A_{22} e^{2 f} d r^{\prime} \int_{a}^{r^{\prime}} A_{11} e^{-2 f} \Phi_{1}\left(\begin{array}{c}
r^{\prime \prime} \\
a, a
\end{array}\right) d r^{\prime \prime}, \\
& \Phi_{2}\left(\begin{array}{c}
r \\
a, a
\end{array}\right)=1-\int_{a}^{r} A_{11} e^{-2 f} d r^{\prime} \int_{a}^{r^{\prime}} A_{22} e^{2 f} \Phi_{2}\left(\begin{array}{c}
r^{\prime \prime} \\
a, a
\end{array}\right) d r^{\prime \prime} .
\end{aligned}
$$

The regular solution at $r=0$ is obtained from (8), if we put $\lambda_{2}=0$ and $a=0$, i.e.:

$$
\begin{aligned}
& C_{1}(r)=e^{-f(r)} \Phi_{1}\left(\begin{array}{c}
r \\
0,0
\end{array}\right) \\
& C_{2}(r)=e^{f(r)} \int_{0}^{r} A_{11} e^{-2 f} \Phi_{1}\left(\begin{array}{c}
r^{\prime} \\
0,0
\end{array}\right) d r^{\prime} .
\end{aligned}
$$

Finally we get:

$$
\begin{aligned}
& g_{v}=u_{1} e^{-f} \Phi_{1}\left(\begin{array}{c}
r \\
0,0
\end{array}\right)+u_{2} e^{f} \int_{0}^{r} A_{11} e^{-2 f} \Phi_{1}\left(\begin{array}{c}
r^{\prime} \\
0,0
\end{array}\right) d r^{\prime} \\
& f_{v}=v_{1} e^{-f} \Phi_{1}\left(\begin{array}{c}
r \\
0,0
\end{array}\right)+v_{2} e^{\ddagger} \int_{0}^{r} A_{11} e^{-2 f} \Phi_{1}\left(\begin{array}{c}
r^{\prime} \\
0,0
\end{array}\right) d r^{\prime}
\end{aligned}
$$

For the existence of the solution (11), or (12), we have only to consider the convergence of the central function $\Phi_{1}\left(\begin{array}{c}r \\ 0,0\end{array}\right)$. The last is guaranteed by the condition $[1]$ :

$$
\bar{q}=\int_{0}^{\infty}\left|A_{22} e^{2 f}\right| d r^{\prime} \int_{0}^{r^{\prime}}\left|A_{11} e^{-2 f}\right| d r^{\prime \prime}<\infty .
$$

If the potential $V(r)$ fullfils the conditions (1) then the function

$$
f(r)=\int_{0}^{r} A_{12} d r^{\prime}
$$


is bounded for any $0 \leqq r \leqq \infty$. Let be $|\not|<\mu$, then we have

$$
\begin{aligned}
\bar{q} & \leqq e^{4 \mu} \int_{0}^{\infty}\left|A_{22}\right| d r \int_{0}^{r}\left|A_{11}\right| d r^{\prime} \\
& \leqq e^{4 \mu} \int_{0}^{\infty}|V|\left\{\left|u_{2}^{2}\right|+\left|v_{2}^{2}\right|\right\} d r \int_{0}^{r}|V|\left\{\left|u_{1}^{2}\right|+\left|v_{1}^{2}\right|\right\} d r^{\prime} .
\end{aligned}
$$

The r.h.s. consists of four terms. If we apply for each of them the argument used in [2] § III we prove that all of them are bounded, provided that the potential $V(r)$ obeys conditions (1).

\section{Results and Discussion}

(i) The application of the integro-iteration method leads, also in the present case, to the explicit expressions of the radial wave functions in a very simple way.

(ii) For $E^{2}-m^{2}=k^{2}>0$ (scattering problems) we find for the phase shifts $\eta_{v}$ :

$$
\tan \eta_{v}=+\frac{e^{f(x)} \int_{0}^{\infty} A_{11} e^{-2 f} \Phi_{1}\left(\begin{array}{c}
r \\
0,0
\end{array}\right) d r}{e^{-f(x)} \Phi_{1}\left(\begin{array}{c}
\infty \\
0,0
\end{array}\right)}
$$

where $v=l$ or $-l-1$. It is understood that for every case we have to employ, for the calculations of $f, A_{11}$ and $A_{22}$, the corresponding expressions of $u_{j}, v_{j}(j=1,2)$ given in the Appendix.

(iii) On the other hand if $E^{2}-m^{2}=-\kappa^{2}<0$ (bound states) we find the eigenvalue equation:

$$
\Phi_{1}\left(\begin{array}{c}
\infty \\
0,0
\end{array}\right)=0 .
$$

(iv) The phase function [6] also is explicitly obtained:

$$
S(r)=+\frac{e^{f(r)} \int_{0}^{r} A_{11} e^{-2 f} \Phi_{1}\left(\begin{array}{c}
r^{\prime} \\
0,0
\end{array}\right) d r^{\prime}}{e^{-f(r)} \Phi_{1}\left(\begin{array}{c}
r \\
0,0
\end{array}\right)} .
$$

It is easy to verify that the phase function (15) is the solution of the Riccati equation:

or,

$$
\begin{aligned}
& S^{\prime}=\left[A_{11}+2 A_{12} S+A_{22} S^{2}\right] \\
& S^{\prime}=V\left[\left(u_{1}+u_{2} S\right)^{2}+\left(v_{1}+v_{2} S\right)^{2}\right]
\end{aligned}
$$

with

$$
S(0)=0 \text {. }
$$


This expression is found in [6]. The difference is due to the different "normalization" of $u_{i}, v_{i}$ which we adopted in order to have

(v) From (12) we find:

$$
\operatorname{det}\left|\begin{array}{ll}
u_{1} & u_{2} \\
v_{1} & v_{2}
\end{array}\right|=1 \text {. }
$$

$$
\begin{aligned}
& \int_{0}^{\infty} V\left(g_{v} u_{1}+f_{v} v_{1}\right) d r=\int_{0}^{\infty} A_{11} e^{-f} \Phi_{1}\left(\begin{array}{c}
r \\
0,0
\end{array}\right) d r \\
+ & \int_{0}^{\infty} A_{12} e^{f} d r \int_{0}^{r} A_{11} e^{-2 f} \Phi_{1}\left(\begin{array}{c}
r^{\prime} \\
0,0
\end{array}\right) d r^{\prime} .
\end{aligned}
$$

Integrating by parts the second term in the r.h.s. we find:

$$
\int_{0}^{\infty} V\left(g_{v} u_{1}+f_{v} v_{1}\right) d r=e^{f(x)} \int_{0}^{\infty} A_{11} e^{-2 \Varangle} \Phi_{1}\left(\begin{array}{c}
r \\
0,0
\end{array}\right) d r .
$$

In a similar way we find:

$$
\int_{0}^{\infty} V\left(g_{v} u_{2}+f_{v} v_{2}\right) d r=-e^{-f(\infty)} \Phi_{1}\left(\begin{array}{c}
\infty \\
0,0
\end{array}\right)+1 .
$$

From (17) and (18) we have:

$$
\tan \eta_{v}=\frac{\int_{0}^{\infty} V\left(g_{v} u_{1}+f_{v} v_{1}\right) d r}{1-\int_{0}^{\infty} V\left(g_{v} u_{2}+f_{v} v_{2}\right) d r}
$$

with $v=l$ or $-l-1$.

The expression (19) is analogeous to that given by Parzen [7], Eq. (71).

(vi) Finally we mention that the method can be applied with the same easiness to the scattering by a modified Coulomb field and it could be useful for the determination of the nuclear charge density $\varrho(r)$ and the corresponding formfactors [5].

\section{Appendix}

If we put in (4) $V=0$ we obtain:

$$
\begin{aligned}
(E+m) f_{v}^{0}+g_{v}^{0 \prime}-\frac{v+1}{r} g_{v}^{0} & =0 \\
-(E-m) g_{v}^{0}+f_{v}^{0 \prime}+\frac{v+1}{r} f_{v}^{0} & =0 .
\end{aligned}
$$


The system can be reduced to two uncoupled Bessel differential equations. If $u_{1}, u_{2}$ correspond to $g_{v}^{0}$ and $v_{1}, v_{2}$ to $f_{v}^{0}$ we make the following choice of the solutions:

(i) $v=l, E^{2}-m^{2}=k^{2}>0$

$$
\begin{array}{ll}
u_{1}=(E+m)^{\frac{1}{2}} \sqrt{\frac{\pi r}{2}} J_{l+\frac{1}{2}}(k r), & v_{1}=(E-m)^{\frac{1}{2}} \sqrt{\frac{\pi r}{2}} J_{l+\frac{3}{2}}(k r), \\
u_{2}=-(E+m)^{\frac{1}{2}} \sqrt{\frac{\pi r}{2}} Y_{l+\frac{1}{2}}(k r), & v_{2}=-(E-m)^{\frac{1}{2}} \sqrt{\frac{\pi r}{2}} Y_{l+\frac{3}{2}}(k r) .
\end{array}
$$

(ii) $v=-l-1, E^{2}-m^{2}=k^{2}>0$

$$
\begin{array}{ll}
u_{1}=(E+m)^{\frac{1}{2}} \sqrt{\frac{\pi r}{2}} J_{l+\frac{1}{2}}(k r), & v_{1}=-(E-m)^{\frac{1}{2}} \sqrt{\frac{\pi r}{2}} J_{l-\frac{1}{2}}(k r), \\
u_{2}=-(E+m)^{\frac{1}{2}} \sqrt{\frac{\pi r}{2}} Y_{l+\frac{1}{2}}(k r), & v_{2}=(E-m)^{\frac{1}{2}} \sqrt{\frac{\pi r}{2}} Y_{l-\frac{1}{2}}(k r) .
\end{array}
$$

(iii) $v=l, E^{2}-m^{2}=-\kappa^{2}<0$

$$
\begin{array}{ll}
u_{1}=(m+E)^{\frac{1}{2}} r^{\frac{1}{2}} I_{l+\frac{1}{2}}(\kappa r), & v_{1}=-(m-E)^{\frac{1}{2}} r^{\frac{1}{2}} I_{l+\frac{3}{2}}(\kappa r), \\
u_{2}=(m+E)^{\frac{1}{2}} r^{\frac{1}{2}} K_{l+\frac{1}{2}}(\kappa r), & v_{2}=(m-E)^{\frac{1}{2}} r^{\frac{1}{2}} K_{l+\frac{3}{2}}(\kappa r) .
\end{array}
$$

(iv) $v=-l-1, E^{2}-m^{2}=-\kappa^{2}<0$

$$
\begin{array}{ll}
u_{1}=(m+E)^{\frac{1}{2}} r^{\frac{1}{2}} I_{l+\frac{1}{2}}(\kappa r), & v_{1}=-(m-E)^{\frac{1}{2}} r^{\frac{1}{2}} I_{l-\frac{1}{2}}(\kappa r), \\
u_{2}=(m+E)^{\frac{1}{2}} r^{\frac{1}{2}} K_{l+\frac{1}{2}}(\kappa r), & v_{2}=(m-E)^{\frac{1}{2}} r^{\frac{1}{2}} K_{l-\frac{1}{2}}(\kappa r) .
\end{array}
$$

With this choice the couples $\left(u_{i}, v_{i}\right)$ satisfy Eqs. (A.1) and, [8]

$$
\operatorname{det}^{-}\left|\begin{array}{cc}
u_{1} & u_{2} \\
v_{1} & v_{2}
\end{array}\right|=1 \text {. }
$$

\section{References}

1. Kanellopoulos, E.J., Kanellopoulos,Th.V., Wildermuth,K.: Commun. math. Phys. 24, 225 (1972).

2. - - Commun. math. Phys. 24, 233 (1972).

3. Darwin, C. G.: Proc. Roy. Soc. A 118, 654 (1928).

4. Mott, N. F.: Proc. Roy. Soc. A 124, 426 (1929).

5. - Massey, H. S. W.: The theory of atomic collisions: $3^{\text {rd }}$ Ed. Ch. IX. Oxford: Clarendon Press 1965. 
6. Ronveaux, A.: Am. J. Phys. 37, 135 (1969).

7. Parzen, G.: Phys. Rev. 80, 261 (1950).

8. Bateman Project: Higher transcendental functions, Vol. II, Ch. VII. New York: McGraw Hill Book Co. Inc. 1953.

\author{
E. J. Kanellopoulos \\ Th. V. Kanellopoulos \\ K. Wildermuth \\ Institut f. Theoretische Physik \\ Universität Tübingen \\ D-7400 Tübingen, Köstlinstr. 6 \\ Germany
}

